ARTICLE

\title{
Coherent control of asymmetric spintronic terahertz emission from two-dimensional hybrid metal halides
}

Kankan Cong ${ }^{1,12}$, Eric Vetter 2,3,12, Liang Yan (3,4,12, Yi Li5 ${ }^{5,6}$, Qi Zhang ${ }^{1,11}$, Yuzan Xiong 5,7, Hongwei Qu', Richard D. Schaller (1) ${ }^{8}$, Axel Hoffmann ${ }^{6,9}$, Alexander F. Kemper (D) 2, Yongxin Yao (1) ${ }^{10}$, Jigang Wang ${ }^{10}$,

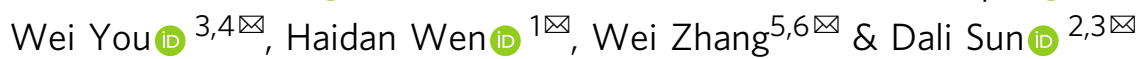

Next-generation terahertz $(\mathrm{THz})$ sources demand lightweight, low-cost, defect-tolerant, and robust components with synergistic, tunable capabilities. However, a paucity of materials systems simultaneously possessing these desirable attributes and functionalities has made device realization difficult. Here we report the observation of asymmetric spintronic- $\mathrm{THz}$ radiation in Two-Dimensional Hybrid Metal Halides (2D-HMH) interfaced with a ferromagnetic metal, produced by ultrafast spin current under femtosecond laser excitation. The generated $\mathrm{THz}$ radiation exhibits an asymmetric intensity toward forward and backward emission direction whose directionality can be mutually controlled by the direction of applied magnetic field and linear polarization of the laser pulse. Our work demonstrates the capability for the coherent control of $\mathrm{THz}$ emission from 2D-HMHs, enabling their promising applications on the ultrafast timescale as solution-processed material candidates for future $\mathrm{THz}$ emitters.

\footnotetext{
${ }^{1}$ Advanced Photon Source, Argonne National Laboratory, Argonne, IL 60439, USA. ${ }^{2}$ Department of Physics, North Carolina State University, Raleigh, NC 27695, USA. ${ }^{3}$ Organic and Carbon Electronics Lab (ORaCEL), North Carolina State University, Raleigh, NC 27695, USA. ${ }^{4}$ Department of Chemistry, University of North Carolina at Chapel Hill, Chapel Hill, NC 27599, USA. ${ }^{5}$ Department of Physics, Oakland University, Rochester, MI 48309, USA. ${ }^{6}$ Materials Science Division, Argonne National Laboratory, Argonne, IL 60439, USA. ${ }^{7}$ Department of Electronic and Computer Engineering, Oakland University, Rochester, MI 48309, USA. ${ }^{8}$ Center for Nanoscale Materials, Argonne National Laboratory, Argonne, IL 64039, USA. ${ }^{9}$ Department of Materials Science and Engineering, University of Illinois at Urbana-Champaign, Urbana, IL 61801, USA. ${ }^{10}$ Ames Laboratory and Department of Physics and Astronomy, lowa State University, Ames, IA 50011, USA. ${ }^{11}$ Present address: Department of Physics, Nanjing University, 210093 Nanjing, P. R. China. ${ }^{12}$ These authors contributed

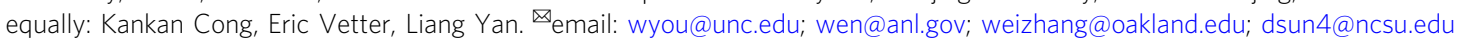


T erahertz $(\mathrm{THz})$ technologies hold great promise for the development of advanced imaging, sensing, security, and communication applications ${ }^{1-3}$. Spintronic-THz, an alternative route for producing $\mathrm{THz}$ radiation using ultrafast spintronics, has recently emerged as a prominent field in conjunction with magnetism, photonics, and ultrafast electro-optics ${ }^{1}$. It holds vast technological advantages that potentially outperform current $\mathrm{THz}$ emitters based on nonlinear optics in terms of ultrabroad bandwidth (e.g., $1-30 \mathrm{THz})^{4,5}$, ultrafast phase control ${ }^{6}$, light-wave acceleration of long-lived currents ${ }^{7,8}$, scalability, and $\operatorname{cost}^{2}$. The seminal works on spintronic- $\mathrm{THz}$ emitters using ferromagnet (FM)/nonmagnetic metallic heterostructures, which convert laser-generated, ultrafast spin current bursts into $\mathrm{THz}$ pulses via the bulk inverse spin Hall effect (ISHE) ${ }^{9}$ (Fig. 1a), helped drive the exploration of $\mathrm{THz}$ generation at Rashba interfaces between two nonmagnetic materials (e.g., two-dimensional electron gas, $2 \mathrm{DEG}$ ) via the inverse Rashba-Edelstein effect (IREE) ${ }^{10-12}$. The ultrafast electric current, $\mathbf{J}_{c}$, that produces the transient $\mathrm{THz}$ emission carried by an interfacial state can be described by:

$$
\mathbf{J}_{c} \propto \lambda_{\text {IREE }} \mathbf{J}_{s} \times \mathbf{s}
$$

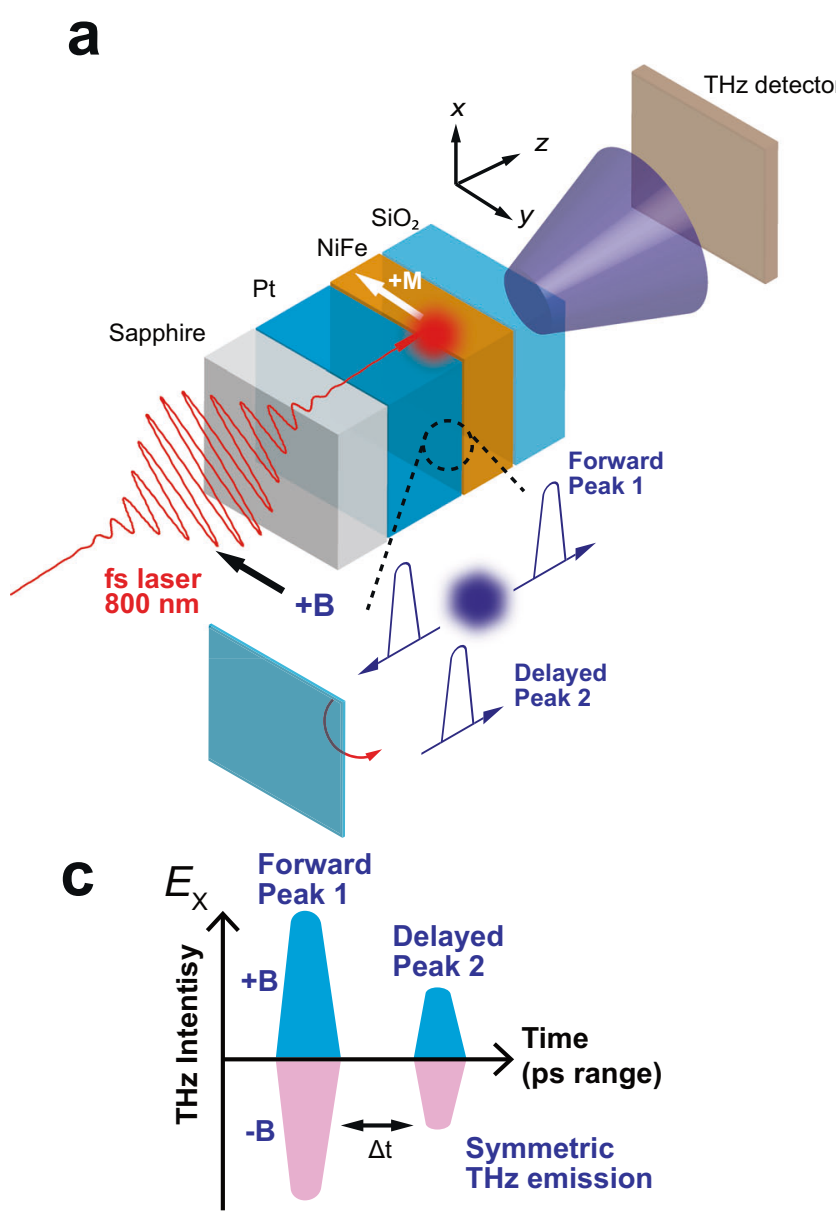

where $\lambda_{\text {IREE }}$ is the spin-to-charge conversion coefficient, also known as the IREE length in the $2 \mathrm{D} \mathrm{limit}{ }^{12}$, $\mathbf{s}$ is the direction of the injected spin polarization parallel to the Rashba interface, and $\mathbf{J}_{s}$ is the femtosecond laser-generated superdiffusive spin current from the adjacent ferromagnetic material (see Fig. 1a). Since an efficient spin-to-charge conversion benefits from the $2 \mathrm{D}$ limit of the cross-section at a Rashba state ${ }^{13}$, the recent demonstration of spintronic- $\mathrm{THz}$ emitters using the $\mathrm{Bi} / \mathrm{Ag} 2 \mathrm{DEG}$ motivates materials research to harness interfacial Rashba systems as efficient spintronic- $\mathrm{THz}$ sources ${ }^{5,6}$.

Two-Dimensional Hybrid Metal Halides (2D-HMHs), a rising star in synthetic semiconductors prepared by cost-effective, lowtemperature solution processing, have recently attracted much research interest ${ }^{14-16}$. $2 \mathrm{D}$-HMHs have been shown to allow for facile and economical, solution-based crude synthesis while still maintaining high energy conversion efficiency, versatile chemical flexibility, quantum well and dielectric confinement effects, and exceptional defect tolerance ${ }^{17}$. These traits have initiated a myriad of interdisciplinary device applications in 2D-HMHs beyond their revolutionary success in photovoltaics ${ }^{18}$. However, their rich spintronic functionalities have yet to be utilized, despite

\section{b}
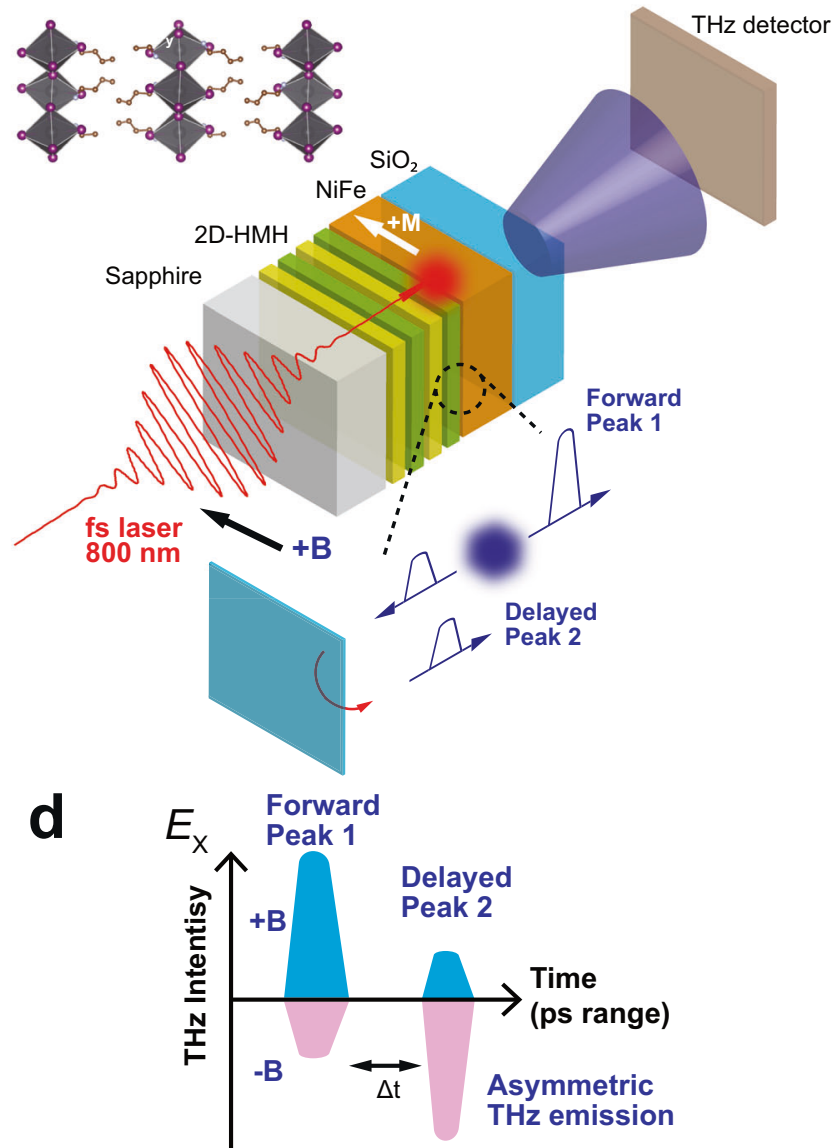

Fig. 1 Schematic illustration of spintronic-THz emission in Pt/NiFe and 2D-HMH/NiFe heterostructures. a A sketch of metallic-based spintronic-THz emission in a Sapphire/Pt/NiFe heterostructure. Under fs laser excitation, the superdiffusive spin current injects into the nonmagnetic metallic layer (e.g., $\mathrm{Pt}$ ) and produces substantial THz radiation via an efficient spin-to-charge conversion process. Generated THz radiation propagates toward the forward $(+z$, i.e., forward peak 1$)$ and backward $(-z)$ direction evenly. The backward THz radiation is reflected at the interface between the sapphire substrate and air, generating a delayed $\mathrm{THz}$ signal (delayed peak 2) collected by the $\mathrm{THz}$ detector $\left(\Delta \mathrm{t}=\mathrm{t}_{1}-\mathrm{t}_{2} \sim 10-11\right.$ picoseconds). $\mathbf{b}$ Schematic structure of the hybrid spintronic- $\mathrm{THz}$ emitter using layered 2D-HMH materials and magnetic field-dependent $\mathrm{THz}$ radiation from the metallic spintronic- $\mathrm{THz}$ emitters along the $\mathrm{x}$-direction in the picosecond timescale. The phase of THz emission depends on the magnetization of the NiFe. $\mathbf{c}, \mathbf{d}$ The illustrated symmetric and asymmetric $\mathrm{THz}$ radiation under fs laser excitation, respectively. An asymmetric $\mathrm{THz}$ radiation emits toward $+z$ and $-z$ direction, of which the intensity is controlled by the applied external magnetic field. The top-left panel in $\mathbf{b}$ shows the schematic crystal structure of prototypical 2D-HMH materials, i.e., twodimensional $(\mathrm{BA})_{2} \mathrm{Pbl}_{4}$. 
theoretical predictions and a handful of pioneering investigations on their large spin-orbit interaction and emergent Rashba state $^{19-22}$. The 2D layered HMHs naturally form 'quantum welllike' structures consisting of a self-assembled periodic array of inorganic perovskite layers, constituting corner sharing $\mathrm{PbI}_{6}$ octahedral slabs (acting as 'wells') separated by organic spacers (acting as 'barriers') in their lattice framework. In contrast to the bulky organic cations composed of only light elements, the periodically layered inorganic $\mathrm{PbI}_{6}$ frameworks dominate the spinorbit coupling of this material (Fig. 1b, inset), suggesting that layered Rashba states may offer an intriguing spin-to-charge conversion in contrast to that from the single-layer Rashba interface in 2DEGs. The combination of these properties stimulates interest in developing solution-processed, hybrid spin-tocharge transducers on the femtosecond timescale using $2 \mathrm{D}-\mathrm{HMH}$ materials for a variety of photonic and $\mathrm{THz}$ applications ${ }^{23}$.

Here we present the report of asymmetric spintronic- $\mathrm{THz}$ emission in 2D-HMHs/ferromagnet heterostructures at room temperature. We show that the emitted $\mathrm{THz}$ electric fields can be generated by an ultrafast, transient spin current pulse from the thin, adjacent FM layer (NiFe) followed by spin-to-charge conversion at femtosecond timescales in $2 \mathrm{D}-\mathrm{HMH}$ materials (Fig. 1b). We find that upon reversing the external magnetic field polarity, both the phase and emission intensity of the THz electric field can be coherently controlled (Fig. 1b), in contrast to that in metallic heterostructures and 3D-HMH materials where the emitted $\mathrm{THz}$ field intensity is mostly independent of the magnetization direction and laser polarization. Our work shows that 2D-HMHs would be desirable material candidates for spintronic$\mathrm{THz}$ generation and manipulation. 2D-HMH materials may prove superior to current conventional semiconductor materials for $\mathrm{THz}$ applications, which require sophisticated deposition approaches that are more susceptible to defects. The improved stability and scalable thin-film process of the reduceddimensional HMH materials enable us to meet the emerging needs for low-cost $\mathrm{THz}$ sources with coherent control capabilities.

\section{Results}

Hybrid spintronic-THz emitter fabrication. Figure $1 \mathrm{~b}$ illustrates the principle of operation and schematic device structure of the hybrid spintronic-THz emitter using $2 \mathrm{D}-\mathrm{HMH}$ materials. The devices are in the form of thin-film multilayers constituting: transparent sapphire substrate $/ \mathrm{BA}_{2} \mathrm{PbI}_{4}(\mathrm{BA}=$ butylammonium $) /$ $\mathrm{NiFe}(5 \mathrm{~nm}) / \mathrm{SiO}_{2}(50 \mathrm{~nm})$ (see Methods and S.I. Fig. S1). The hybrid $\mathrm{THz}$ emitters based on these polycrystalline $2 \mathrm{D}-\mathrm{HMH}$ thin films are prepared on double-polished sapphire substrates by a lowtemperature solution-processed spin coating approach (S.I. Fig. S1). The thickness of the thin film is kept above $100 \mathrm{~nm}$ to ensure a smooth and uniform film morphology for the following metal deposition. The quality of the prepared thin film is checked by atomic force microscopy, showing a root mean square (RMS) roughness of $\sim 1.3 \mathrm{~nm}$ (S.I. Fig. S2). The layered perovskite crystal structures are validated by X-ray diffraction and are consistent with the literature reports ${ }^{24}$. The optical absorption measurement shows distinctive absorption peaks in the films depending on the quantum well confinement formed between inorganic metal halides and bulky organic ligands ${ }^{24}$. Negligible absorption at $800 \mathrm{~nm}$ suggests that the below-gap laser excitation directly interacts with the ferromagnetic layer (i.e., $\mathrm{NiFe}$ ) without an intensity loss from passing through the 2D-HMH layer in the current device configuration. A flat response of the circular dichroism spectra confirms that there is no indication of chiral structure formation, or negligible preferred circular polarization absorption (S.I. Fig. S3).

Upon linearly polarized fs laser excitation, a superdiffusive spin current is generated in the NiFe layer ${ }^{25}$ and, in turn, is injected into the layered 2D-HMH thin film causing the emission of $\mathrm{THz}$ radiation via ultrafast spin-to-charge conversion. The generated $\mathrm{THz}$ radiation was recorded through the electro-optical sampling technique ${ }^{26}$. The set of unit vectors $x, y$, and $z$ represent the chosen coordinate system. A pair of wire-grid polarizers are used to measure the parallel (i.e., $E_{y} \perp B_{y}$ ) and perpendicular (i.e., $E_{x} \| B_{y}$ ) components of $\mathrm{THz}$ radiation (see Methods) with respect to the direction of the in-plane magnetic field, $B_{\mathrm{y}} . B_{y}$ was applied by using a reversible two-pole permanent magnet at a constant magnetic field of $\sim 500$ Oe, which is adequately large to maintain the magnetization of the NiFe layer along the in-plane direction (i.e., $y$-direction) during $\mathrm{THz}$ measurements.

Magnetic Field dependence of asymmetric $\mathrm{THz}$ radiation. Figure 2a presents the typical measured $\mathrm{THz}$ signal $E_{x}(t)$ in $2 \mathrm{D}$ hybrid spintronic- $\mathrm{THz}$ emitters at room temperature. Each $\mathrm{THz}$ transient is fully inverted when the polarity of the magnetic field is reversed, confirming a spintronic origin: Since the $\mathrm{THz}$ signal is produced by the superdiffusive spin current burst generated by the NiFe layer, switching the direction of the magnetization of the NiFe layer reverses the spin polarization of the injected spin current. Consequently, the $\mathrm{THz}$ signal polarity (i.e., $\mathrm{THz}$ phase) from the $2 \mathrm{D}-\mathrm{HMH}$ thin film is $180^{\circ}$ shifted. The magnetic fieldinduced phase shift confirms the ultrafast spin-to-charge conversion in $2 \mathrm{D}-\mathrm{HMH}$ thin films.

Following the $\mathrm{THz}$ generation mechanism in the metallic spintronic- $\mathrm{THz}$ emitters ${ }^{3}$, the $\mathrm{THz}$ radiation will emit toward both $-z$ (backward) and $+z$ (forward) directions. Therefore, all measured time traces of the $\mathrm{THz}$ signal contain two peak groups. The first one is the forward THz radiation (labeled as 'peak 1' appeared at $\mathrm{t}_{1}=\sim 5 \mathrm{ps}$ ) and the delayed one is the backward $\mathrm{THz}$ radiation (labeled as the delayed 'peak 2' appeared at $\mathrm{t}_{2}=\sim 15 \mathrm{ps}$ ) due to the reflection at the interface between the sapphire substrate and the air (Fig. 1b). The time difference ( $\Delta \mathrm{t}=\sim 10-11 \mathrm{ps}$ ) between the two peak groups matches the time delay required for the backward $\mathrm{THz}$ pulse to transmit through the $0.5 \mathrm{~mm}$-thick sapphire substrate twice (refractive index: 3.4). Strikingly, we found the presence of a nontrivial asymmetry in $\mathrm{THz}$ intensity in both peak groups depending on the direction of the applied magnetic field. For the first peak group, the intensity of the forward $\mathrm{THz}$ radiation at the positive magnetic field $(+\mathbf{B}$, blue trace) is roughly two times larger than the one at $-\mathbf{B}$. In contrast, the second peak group (i.e., backward $\mathrm{THz}$ radiation) exhibits an asymmetry: THz intensity at $+\mathbf{B}$ is clearly weaker than the one at $-\mathbf{B}$. This reversed asymmetry of the THz intensity suggests fielddependent asymmetric $\mathrm{THz}$ radiation along $-z$ (backward) and $+z$ (forward) directions (see also the illustration in Fig. 1b).

To verify that the observed asymmetric $\mathrm{THz}$ emission is uniquely present in $2 \mathrm{D}-\mathrm{HMH} / \mathrm{NiFe}$ samples, we have conducted spintronic- $\mathrm{THz}$ measurements in a series of control samples including $\mathrm{NiFe} / \mathrm{Pt}$ and bare NiFe films (see Fig. 2b). None of them exhibits a similar asymmetric $\mathrm{THz}$ intensity at opposite directions of magnetic fields, ruling out the sample/field misalignment during the $\mathrm{THz}$ measurement as a possible source of the observed behavior. Moreover, in all the metallic spintronic- $\mathrm{THz}$ devices, the phases of the $\mathrm{THz}$ radiation for the 1st and 2nd peak group remain the same except for the decreased intensity that has been attributed to the Fabry-Perot process and multiple reflections between the metallic heterostructure and the substrate ${ }^{27}$.

Moreover, the phase of the $\mathrm{THz}$ transient in the 2D-HMH/ NiFe heterostructures between the 1st and 2nd peak group is clearly inverted, although the time delay $(\Delta t=\sim 10-11 \mathrm{ps})$ between the two peak groups is similar to those in other metallic $\mathrm{THz}$ devices. This distinct feature also helps to eliminate the possibility of $\mathrm{THz}$ emission generated by the NiFe itself (which 
a

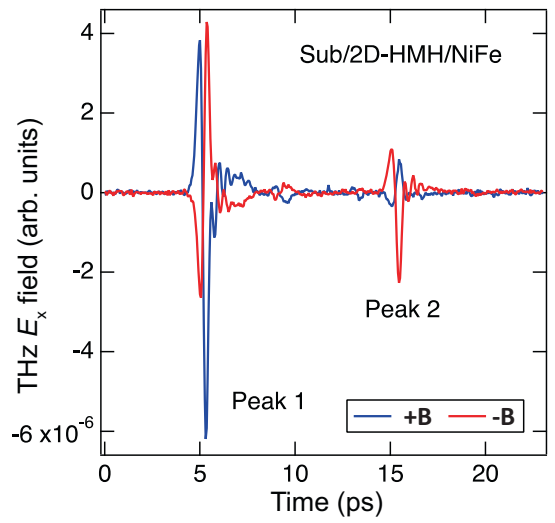

C

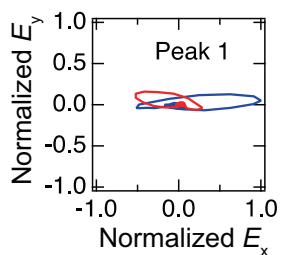

d

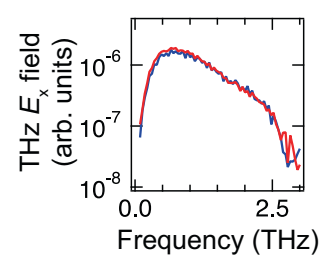

b

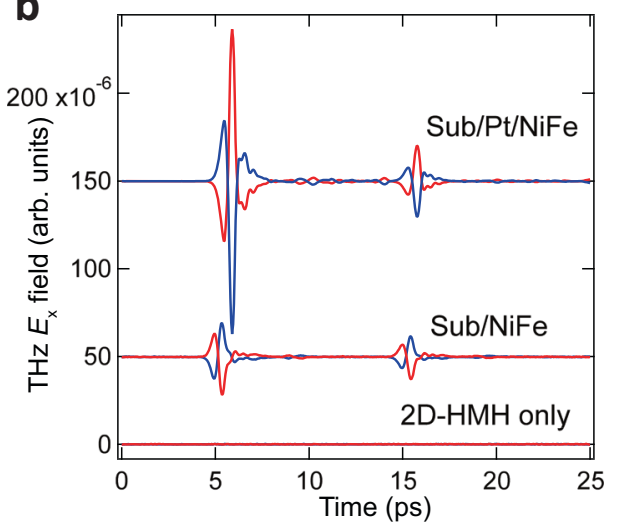

e

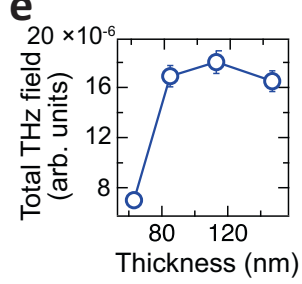

f

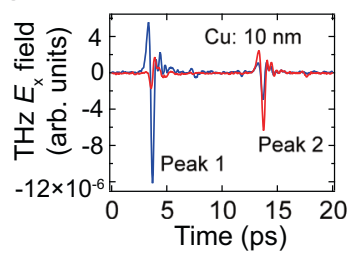

Fig. 2 Magnetic field-dependent asymmetric THz radiation upon linearly polarized laser excitation. a Measured time trace of the THz pulse generated from the $2 \mathrm{D}-\mathrm{HMH} / \mathrm{NiFe}$ heterostructures under positive $(+\mathbf{B})$ and negative $(-\mathbf{B})$ magnetic fields, respectively. The forward (peak 1 at $\left.\mathrm{t}_{1}=\sim 5 \mathrm{ps}\right)$ and backward (peak 2 at $t_{2}=\sim 15 \mathrm{ps)} \mathrm{THz} \mathrm{pulses} \mathrm{exhibit} \mathrm{opposite} \mathrm{symmetry} \mathrm{of} \mathrm{the} \mathrm{intensity} \mathrm{depending} \mathrm{on} \mathrm{the} \mathrm{direction} \mathrm{of} \mathrm{the} \mathrm{applied} \mathrm{magnetic} \mathrm{field.} \mathbf{b}$ Symmetric $\mathrm{THz}$ radiations are confirmed in NiFe/Pt and NiFe only control samples. No THz signal is observed in the 2D-HMH film without the ferromagnet top-layer under the same laser excitation. $\mathbf{c}$ The parametric plot of the emitted THz field shows the THz polarization is mainly along the $x$-direction. $\mathbf{d}$ The $\mathrm{THz}$ emission spectra of $\mathbf{a}$ indicate equal total $\mathrm{THz}$ radiation in spite of asymmetric radiation along with two propagation directions. e $\mathrm{THz}$ signals show a very weak $2 \mathrm{D}-\mathrm{HMH}$ thickness dependence at the higher $2 \mathrm{D}-\mathrm{HMH}$ thicknesses above $\sim 80 \mathrm{~nm}$. The error bars represent the standard errors of the measurements. f Pronounced asymmetric $\mathrm{THz}$ radiation in $2 \mathrm{D}-\mathrm{HMH} / \mathrm{Cu} / \mathrm{NiFe}$ trilayer structure, ruling out the possible proximity effect induced nonreciprocal phenomena at the $2 \mathrm{D}-\mathrm{HMH} / \mathrm{NiFe}$ interface.

should retain identical phases between two peak groups as shown in Fig. 2b), confirming that the dominant mechanism of spintronic- $\mathrm{THz}$ generation stems from the $2 \mathrm{D}-\mathrm{HMH}$ layer via the injected superdiffusive spin current. The observed $\mathrm{THz}$ signal is in contrast to the null $\mathrm{THz}$ signal measured from the substrate/ 2D-HMH/SiO 2 (see Fig. 2b). Under $800 \mathrm{~nm}$ below-gap excitation, we do not observe any measurable $\mathrm{THz}$ emission in the $2 \mathrm{D}$ $\mathrm{HMH} / \mathrm{SiO}_{2}$ sample within our detection capability, excluding the nonspintronic THz emission due to the photo-Dember effect ${ }^{28,29}$, nonlinear optical effect ${ }^{30}$, or linear/circular photogalvanic effect which requires photocarriers under the above-gap laser excitation $^{19}$. Magnetic field effect contributing to the $\mathrm{THz}$ radiation in the $2 \mathrm{D}-\mathrm{HMH}$-only device ${ }^{31}$ is unlikely due to the absence of asymmetric $\mathrm{THz}$ emission under $400 \mathrm{~nm}$ excitation.

A parametric plot of the generated $\mathrm{THz}$ electric-field amplitude for the first peak group is presented in Fig. 2c. The forward $\mathrm{THz}$ radiation is mainly polarized along the $\mathrm{x}$-direction, perpendicular to the magnetization of the NiFe layer (Fig. 1b), denoting the 'spintronic' nature of $\mathrm{THz}$ generation (i.e., $E_{x}(\mathrm{THz}) \propto \mathbf{J}_{s} \times S_{y}$ ). Figure $2 \mathrm{~d}$ shows the Fourier spectra of the $\mathrm{THz}$ transient signal in the $2 \mathrm{D}-\mathrm{HMH} / \mathrm{NiFe}$ heterostructure for both magnetic field directions. While the 1 st and 2 nd peak groups exhibit different intensities, the $+\mathbf{B} /-\mathbf{B}$ traces are roughly aligned with each other suggesting an equal intensity of overall $\mathrm{THz}$ signals stemming from the same amount of superdiffusive spin current in the 2D$\mathrm{HMH}$ film at opposite magnetic fields. The normalized amplitude spectrum of the $\mathrm{THz}$ radiation shows a broader bandwidth compared to the one obtained from the NiFe/Pt (S.I. Fig. S4). The bandwidth goes up to $3 \mathrm{THz}$, which is partly due to the limitation imposed by the use of a $\mathrm{ZnTe}$ crystal in the measurement setup. Figure $2 \mathrm{e}$ shows the observed overall $\mathrm{THz}$ intensity as a function of $2 \mathrm{D}-\mathrm{HMH}$ thickness. The decreased $\mathrm{THz}$ signal below $80 \mathrm{~nm}$ can be understood as a byproduct of poor thin-film quality that results in decreased spin-to-charge conversion at the interface between $\mathrm{NiFe}$ and 2D-HMH layer ${ }^{16}$. The weak thickness dependence of $\mathrm{THz}$ intensity at higher thickness implies that the interfacial spin-to-charge conversion is responsible for the spintronic- $\mathrm{THz}$ generation rather than the bulk-dominated ISHE process. THz signals have additionally been measured in a trilayer device structure constituting substrate/2D-HMH/Cu $(10 \mathrm{~nm}) /$ $\mathrm{NiFe}$ to further separate the $\mathrm{THz}$ signal from the magnetic proximity effect (Fig. 2f). By inserting a thin $\mathrm{Cu}$ spacer layer between the 2D-HMH and NiFe layer, the direct proximity effect between the 2D-HMH and $\mathrm{NiFe}$ is suppressed, while the superdiffusive spin current can still transmit through the $\mathrm{Cu}$ spacer layer and inject into the 2D-HMH layer thanks to the long spin diffusion length of $\mathrm{Cu}^{32}$. A pronounced asymmetric $\mathrm{THz}$ radiation is still observed when the thickness of the $\mathrm{Cu}$ spacer layer is $10 \mathrm{~nm}$. This suggests that the observed asymmetric $\mathrm{THz}$ radiations are not attributed to the magnetic proximity effect ${ }^{33}$.

Sample orientation dependence of asymmetric $\mathrm{THz}$ radiation. To further elucidate the origin of asymmetric $\mathrm{THz}$ radiation in the $2 \mathrm{D}-\mathrm{HMH} / \mathrm{NiFe}$ heterostructure ${ }^{34}$, sample orientation dependence of the $\mathrm{THz}$ electric field is measured as shown in Fig. 3. We conducted the $\mathrm{THz}$ measurements for two orientations of the sample by flipping the same hybrid heterostructure around the applied magnetic field over $180^{\circ}$ so that the incident laser direction was inverted. In Fig. 3a, the laser is incident from the sapphire substrate before successively passing through the 2D$\mathrm{HMH}$ and then the NiFe layers, respectively. Under this condition, the generated spin current was injected back into the 2D-HMH 

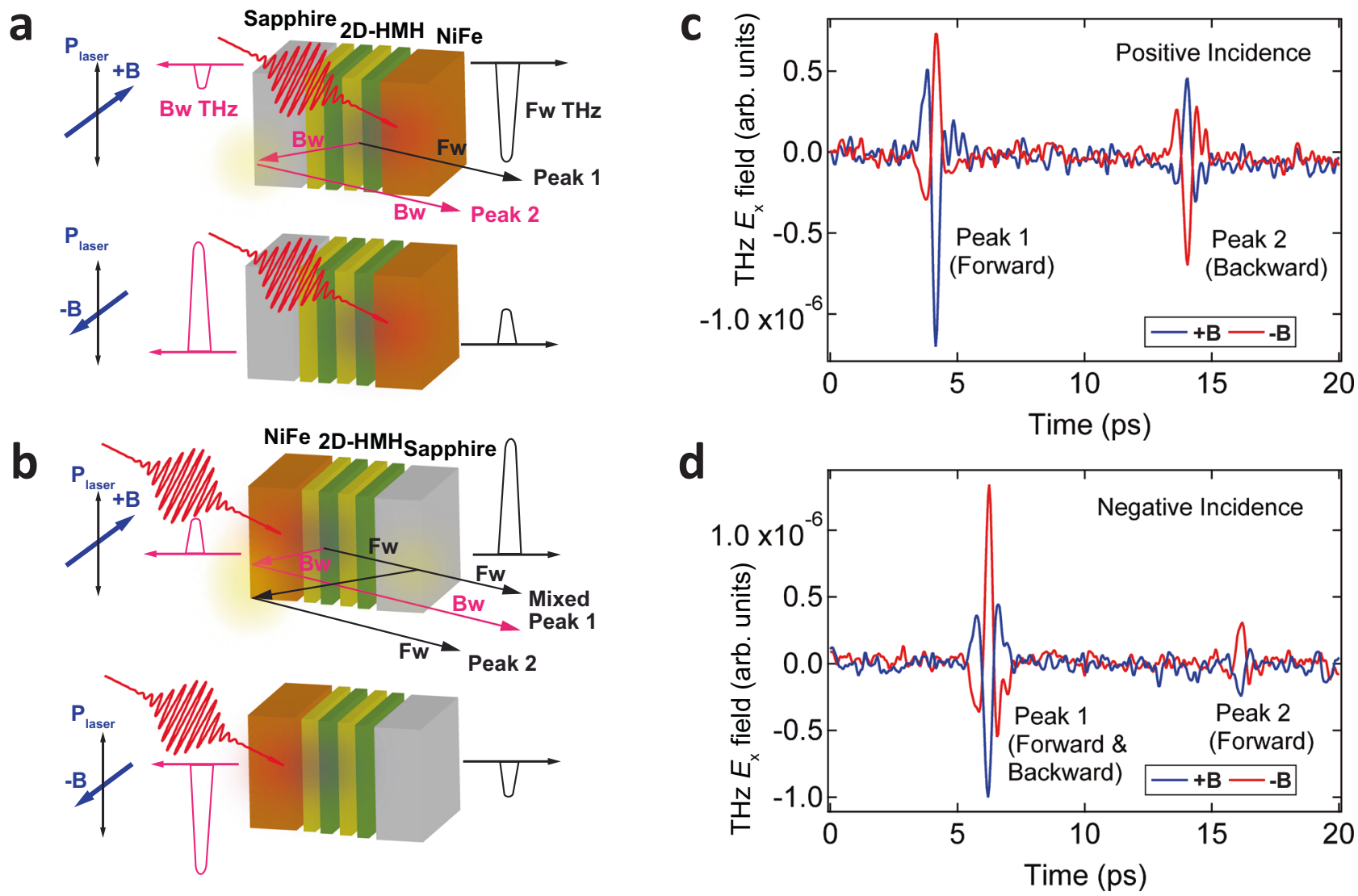

Fig. 3 Sample orientation dependence of asymmetric THz radiation. $\mathbf{a}$, $\mathbf{b}$ Schematic illustration of the $E_{x}$ component of generated THz electric field in $2 \mathrm{D}$ $\mathrm{HMH} / \mathrm{NiFe}$ heterostructure using front and back pump configurations, respectively. The red and blue spots illustrate where the laser pulse is absorbed in the NiFe layer and $\mathrm{THz}$ emission is emitted from the 2D-HMH layer, respectively. The black (pink) solid lines represent the optical paths of the forward (backward) $\mathrm{THz}$ emission inside the device. The yellow spots indicate the positions where the THz emissions are reflected. c, $\mathbf{d}$ Obtained electric field of $\mathrm{THz}$ radiation along the $x$-direction as a function of time for opposite incidence direction of light, showing a clear sample orientation dependence of the directionality of the $E_{\mathrm{x}}$ component: The directionality and phase of the $E_{\mathrm{x}}$ component of THz radiation change their polarity with the magnetization and sample flips, respectively.

film opposite to the incident laser direction. The forward $\mathrm{THz}$ radiation (peak 1$)$ propagates through the $\mathrm{FM}$ layer $(+z$ direction) and the backward peak group ( $-z$ direction, peak 2$)$ propagates through the sapphire substrate twice. This configuration is labeled as 'positive incidence' as the normal condition for our $\mathrm{THz}$ measurement (Fig. 3a). The 'negative incidence' refers to the situation where the laser was incident to the $\mathrm{NiFe}$ layer first. Under this condition, the forward $\mathrm{THz}$ radiation (at $\mathrm{t}_{1}=\sim 6 \mathrm{ps}$ ) will propagate through the sapphire once, shown as the peak 1 . Now the delayed peak 2 (at $t_{2}=\sim 16 \mathrm{ps}$ ) is actually also originated from the forward $\mathrm{THz}$ emission having the same phase as peak 1 because it was reflected at the interface between the air and $0.5 \mathrm{~mm}$-thick sapphire substrate. The backward $\mathrm{THz}$ emission under this condition overlaps with the forward $\mathrm{THz}$ emission at peak 1 since it cannot be solely separated due to the reflection occurred at the interface between the air and $5 \mathrm{~nm}$-thick NiFe layer (see the illustrated optical paths in Fig. 3a and 3b).

Figure $3 \mathrm{c}$ and $\mathrm{d}$ clearly shows that the electric $\mathrm{THz}$ field changes sign upon the reversal of both sample orientation and magnetic field, respectively. The slight change in delay and timescale of the first $\mathrm{THz}$ peak group arises from the different propagation paths of $\mathrm{THz}$ radiation and pump light. When the magnetization is fixed (e.g., at $+\mathbf{B}$ ), turning the sample orientation from the positive to negative incidence geometry does result in an opposite sign of the $\mathrm{THz}$ electric field because of the changed diffusion direction of the spin current $\left(\mathbf{J}_{s}\right)$. However, we found the asymmetric $\mathrm{THz}$ intensity does not change upon the reversal of sample orientation: $\mathrm{THz}$ intensity at $+\mathbf{B}$ is consistently larger than that at $\mathbf{- B}$ regardless of the laser incident direction. Once the angle between the applied magnetic field and linear polarization axis is determined, the asymmetric $\mathrm{THz}$ radiation intensity is kept unchanged, although the phase of the $\mathrm{THz}$ electric field is fully inverted. This indicates that the role of potential structure-induced spontaneous symmetry breaking at the metallic/hybrid semiconductor interface ${ }^{34}$ (a cross product of polar unit vector $\left(\mathbf{n}_{z}\right)$ normal to the interface and the applied field, B) is not responsible for the asymmetric spintronic-THz emission, otherwise the weaker intensity of forward $\mathrm{THz}$ radiation would be expected in the 'negative incidence' configuration at $+\mathbf{B}$. The lack of spontaneous symmetry breaking in the heterostructure is further corroborated by the absence of reversed $E_{\mathrm{y}}$ component under the circularly polarized light illumination (S.I. Fig. S5) ${ }^{34}$.

Coherent control of $\mathrm{THz}$ radiation by pump-light polarization. As suggested by the sample orientation dependence in Fig. 3, the relative orientation between the laser polarization axis and applied magnetic field may play a key role in determining the asymmetric $\mathrm{THz}$ radiation in the hybrid $\mathrm{THz}$ emitter. Thus, pump polarization dependence of the $\mathrm{THz}$ electric field has been measured below. The geometry of the linearly polarized laser 
excitation and $\mathrm{THz}$ detection setup is depicted in Fig. $4 \mathrm{a}$ and $\mathrm{b}$. Figure $4 \mathrm{c}$ and $\mathrm{d}$ show the typical $2 \mathrm{D}$ contour plot of $\mathrm{THz}$ radiation as a function of time and polarization angle, $\Theta$, at the positive and negative magnetic field, respectively. The change of $\mathrm{THz}$ intensity in the 1st and 2nd peak group as a function of linear polarization angle is summarized in Fig. $4 \mathrm{e}$ and $\mathrm{f}$, respectively.

In principle, once the spin polarization and injection direction of the spin current is fixed by the sample structure and magnetization, no dependence of spintronic- $\mathrm{THz}$ intensity on the pump polarization should be expected for the spintronic- $\mathrm{THz}$ emission as recently reported ${ }^{4,35}$. Strikingly, we found that the emitted $\mathrm{THz}$ intensity shows a strong dependence on pump polarization: When applying $+\mathbf{B}$, by rotating the laser polarization axis from 0 to 90 degrees, the amplitude of the forward $\mathrm{THz}$ peak group (blue trace in Fig. 4e) keeps decreasing until it reaches a minimum at $\sim 90$ degrees while the amplitude of the backward peak group increases (blue traces in Fig. 4f) inversely. At $-\mathbf{B}$, the change of $\mathrm{THz}$ amplitude between two peak groups reverse their signs with respect to that at $+\mathbf{B}$. The observed polarizationdependent simultaneous decrease (increase) of $\mathrm{THz}$ amplitude in peak 1 and the increase (decrease) in peak 2 rules out the trivial laser-induced absorption or transmission difference for $\mathrm{THz}$ pulses in the c-cut sapphire substrate, by which the $\mathrm{THz}$ amplitude in both peaks should follow exactly the same trace without any magnetic field control. A large percentage of the $\mathrm{THz}$ amplitude is modulated by the pump polarization up to $30-40 \%$, demonstrating the realization of coherent control of $\mathrm{THz}$ amplitude in the hybrid spintronic- $\mathrm{THz}$ emitter.

Both magnetic field and pump polarization-dependent $\mathrm{THz}$ measurements have been performed in 3D-HMH-based hybrid spintronic- $\mathrm{THz}$ devices, i.e., $\mathrm{CH}_{3} \mathrm{NH}_{3} \mathrm{PbBr}_{3} / \mathrm{NiFe}$. While the $3 \mathrm{D}$ $\mathrm{CH}_{3} \mathrm{NH}_{3} \mathrm{PbBr}_{3}$ presumably possesses a similarly large spin-orbit coupling as that in $2 \mathrm{D}-\mathrm{HMH}$ materials, as well as a surface Rashba state ${ }^{16}$ that can produce similar spintronic- $\mathrm{THz}$ radiation, neither asymmetric $\mathrm{THz}$ radiation nor pump polarization dependence is observed (S.I. Fig. S6). This implies that the observed asymmetric $\mathrm{THz}$ radiation strongly correlates with the low-dimensionality of the 2D-HMH layer under below-gap fs laser excitation.

$\mathrm{THz}$ transmission measurements have been performed in the same $2 \mathrm{D}-\mathrm{HMH} / \mathrm{NiFe}$ heterostructure without fs laser excitation. In the absence of fs laser excitation, there is no detectable difference for the $\mathrm{THz}$ transmission and absorption along both $+z$ and $-z$ directions (S.I. Fig. S7). The THz electric field is also independent of the polycrystalline sample orientation along the in-plane direction, which is corroborated by the magnetic anisotropy measurements, namely rotating the device within the $x-y$ plane while keeping the laser pulse linear polarization $(+x-$ direction) and the magnetic field ( $+y$-direction) constant (S.I. Fig. S8). The measured THz electric-field intensity and polarity shows no difference at different angles, confirming that the asymmetric $\mathrm{THz}$ radiation neither originate from the magnetic anisotropy of the NiFe nor the crystalline structure of the 2D-HMH film and its relative orientation with respect to the laser polarization axis.

\section{Discussion}

To put the above asymmetric $\mathrm{THz}$ emission findings on a stronger footing, we now consider possible mechanisms that may underpin the observed field- and polarization-dependent asymmetric $\mathrm{THz}$ radiation in hybrid spintronic- $\mathrm{THz}$ emitters. The control experiments presented in the previous section are inconsistent with several possible causes (see detailed discussion in S.I. section XI) including field-dependent spin-to-charge conversion at the $3 \mathrm{D}-\mathrm{HMH} / \mathrm{NiFe}$ interface (S.I. Fig. S6), $\mathrm{THz}$ transmission (S.I. Fig. S7), magnetic anisotropy in the NiFe film (S.I. Fig. S8), and the role of $\mathrm{SiO}_{2}$ capping layer (S.I. Fig. S9-S10).

Thus we extend the IREE mechanism by including an additional in-plane momentum shift of the Rashba bands caused by a joint effect of both the applied magnetic field $B$ and timedependent electric polarization induced by linearly polarized fs laser pump pulses, as illustrated in Fig. 5a and b. Considering a typical Rashba band splitting ${ }^{36}$, being symmetric with respect to spin and momentum ${ }^{20}$, we would obtain ultrafast charge current

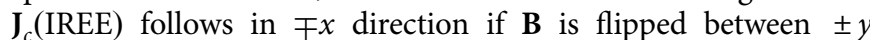
direction due to the IREE. Such a transient charge current flow a

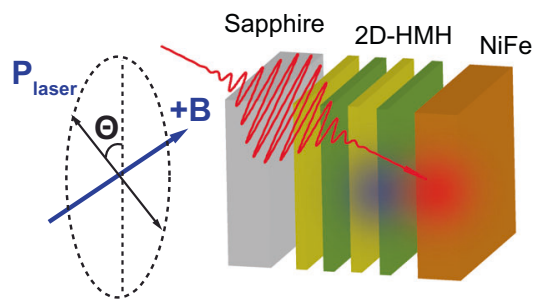

b

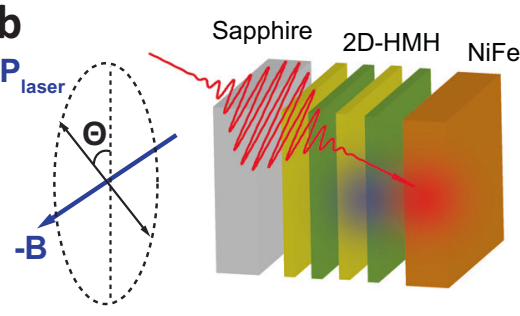

c
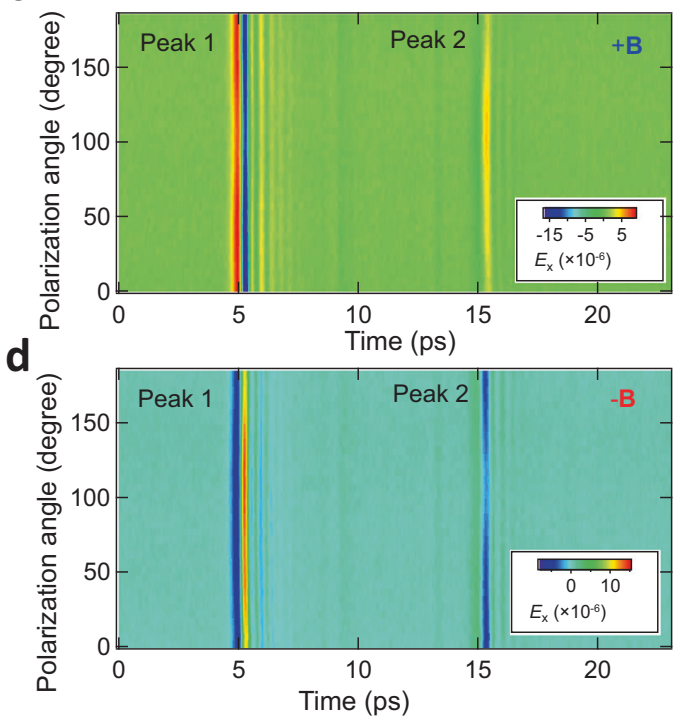

e

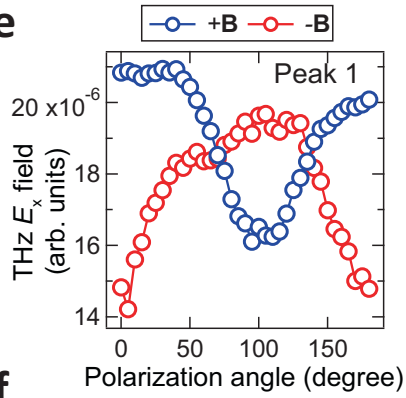

f

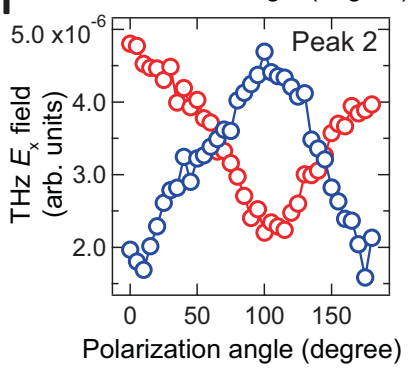

Fig. 4 Pump polarization dependence of asymmetric THz radiation. $\mathbf{a}$, $\mathbf{b}$ Schematic illustration of pump polarization dependence of THz measurement in $2 \mathrm{D}-\mathrm{HMH} / \mathrm{NiFe}$ heterostructure as a function of the relative angle $(\Theta)$ between pump linear polarization axis with respect to the magnetic field. $\mathbf{c}$, $\mathbf{d} 2 \mathrm{D}$ contour plot of the electric field of $\mathrm{THz}$ radiation along the $x$-direction as a function of time and polarization angle $(\Theta)$, exhibiting strong linear polarizationmodulated THz intensity in both two peak groups. Panels $\mathbf{e}$ and $\mathbf{f}$ summarize the opposite changes of the polarization-dependent $\mathrm{THz}$ intensity in Peak 1 and Peak 2 with the switching of the magnetic field, respectively. 

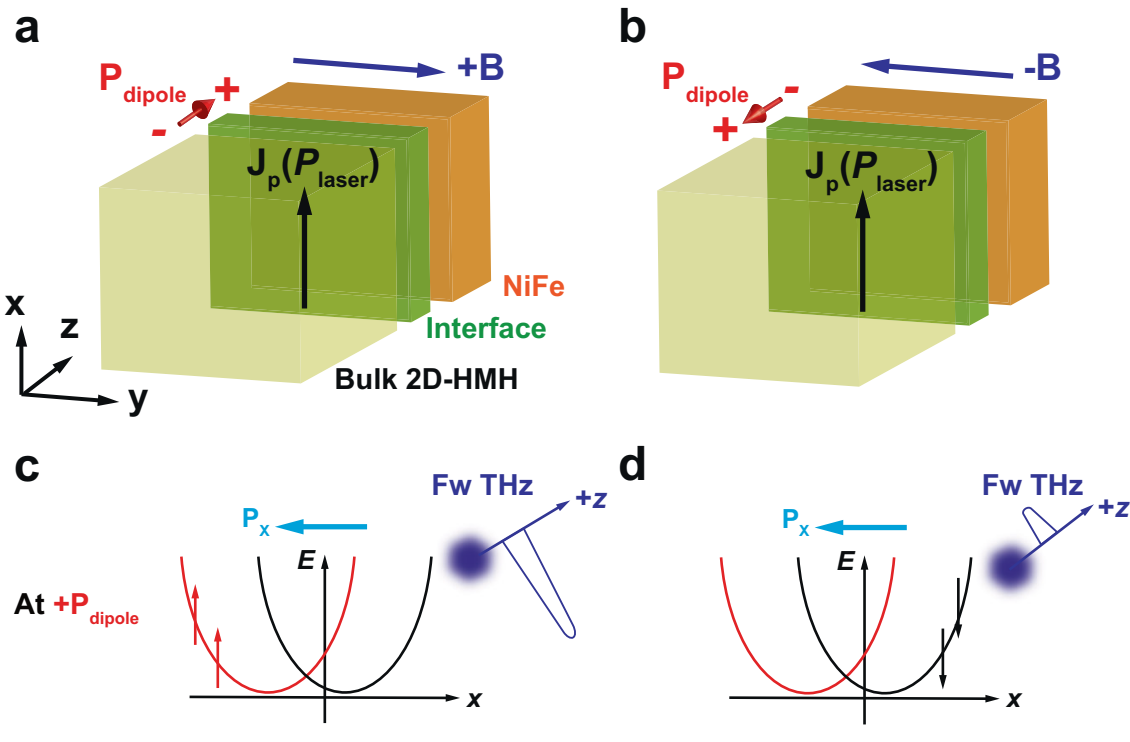

$J_{c}(T H z)=-J_{c}(I R E E)-J_{c}\left(P_{x}\right)$

$J_{c}(T H z)=+J_{c}\left(\right.$ IREE) $-J_{c}\left(P_{x}\right)$
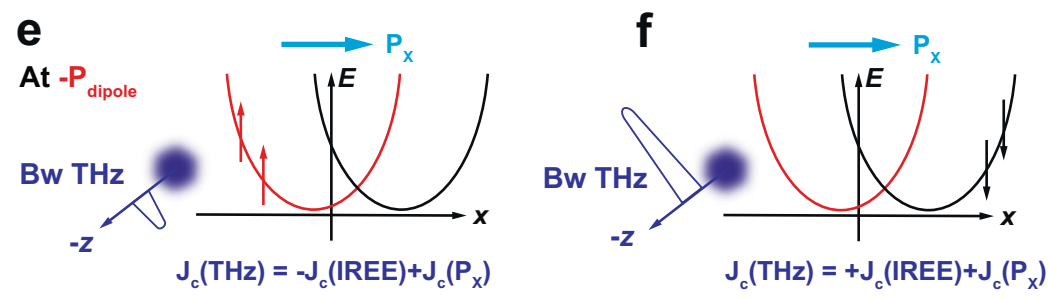

Fig. 5 Schematic illustration of the shifted Rashba bands and resulting asymmetric THz emission. $\mathbf{a}, \mathbf{b}$ Formation of an electric polarization $\mathbf{p}_{\text {dipole }}(z)$ along the $z$-axis across the interfacial layered structure of the $2 \mathrm{D}-\mathrm{HMH}$ film in the vicinity of the $2 \mathrm{D}-\mathrm{HMH} / \mathrm{NiFe}$ interface under positive $(+\mathbf{B})$ and negative ( $-\mathbf{B})$ magnetic fields, respectively. $\mathbf{J}_{p}\left(P_{\text {laser }}\right)$ is the polarization current along the $x$-direction induced by linear polarization of the laser pump pulse. $\mathbf{c}$, $\mathbf{d} A$ sketch of the shifted Rashba bands in the presence of momentum shift, $\mathbf{P}_{x}<0$ at the $+\mathbf{p}_{\text {dipole }}(z)$ side according to Eq. (2). The red (black) arrows indicate the injected spin polarization at $+\mathbf{B}(-\mathbf{B})$ magnetic field. $\mathbf{e}, \mathbf{f}$ The shifted Rashba bands at the $-\mathbf{p}_{\text {dipole }}(z)$ side $\left(\mathbf{P}_{\mathrm{x}}>0\right)$. The generated THz emission containing both the field-dependent IREE and the field-independent momentum shift components is shown at different fields and surfaces that account for the asymmetric $\mathrm{THz}$ radiation.

will in turn only generate symmetric THz emission by flipping $\mathbf{B}$, i.e., an equal amplitude with a $\pi$-phase flip similar to that in $\mathrm{NiFe} /$ $\mathrm{Pt}$ and NiFe only control samples. However, at the 2D-HMH/ $\mathrm{NiFe}$ interface, a polarization current, $\mathbf{J}_{p}\left(P_{\text {laser }}\right)$ can be induced by time-dependent laser polarization $\left(P_{\text {laser }}\right)$ of the pump pulse. The $\mathbf{J}_{p}\left(P_{\text {laser }}\right)$ effect can be significantly amplified at the $2 \mathrm{D}-\mathrm{HMH}$, assisted by the Rashba states, i.e., the $\mathbf{J}_{p}\left(P_{\text {laser }}\right)$ leads to an in-plane momentum shift of the Rashba bands along the $x$-direction, playing a key role in determining the asymmetric contribution to the $\mathrm{THz}$ emission amplitude with respect of the applied $\mathbf{B}$ field as shown in Fig. 2a.

A physical picture of shifted Rashba bands is further elaborated below. Primarily, we describe the origin of the in-plane momentum shift of Rashba bands due to the concerted actions of an electric dipole along the $z$-axis and the ordinary Hall effect. Such behavior can be understood as a consequence of the simultaneous breaking of time-reversal and space-inversion symmetry as typically described in two-dimensional quantum well systems ${ }^{37}$ :

$$
\mathbf{P}_{x}=\mathbf{P}_{C M}-\frac{\mathbf{B}}{c} \times \mathbf{p}_{\text {dipole }}(z)
$$

where the momentum $\mathbf{P}_{x}$ is shifted from original center of mass motion $\mathbf{P}_{C M}$ by an amount that arises from the Lorentz force acting on an electric dipole along the $z$-axis, $\mathbf{p}_{\text {dipole }}(z)=e \mathbf{r}_{z}$. This leads to a rigid in-plane momentum shift of the Rashba bands along the $x$-axis perpendicular to the applied external magnetic field.

The formation of the electric dipole could be understood as the interaction between applied magnetic field and light electric fieldinduced polarization current. The incident pump laser with polarization along the $x$ direction induces a polarization current $\mathbf{J}_{p}\left(P_{\text {laser }}\right)$, which will keep the same direction when the $\mathbf{B}$ field reverses. In analogy to ordinary Hall effect caused by conduction current, such polarization current simultaneously breaks timereversal and space-inversion symmetries in the $2 \mathrm{D}$ quantum well structure, leading to the electric dipole $\mathbf{p}_{\text {dipole }}(z)$ perpendicular to the $\mathbf{B}$ field and light polarization. As illustrated in Fig. $5 \mathrm{a}$ and $\mathrm{b}$, the electric polarization $\mathbf{p}_{\text {dipole }}(z)$ is formed along the $z$-axis across the interfacial layered structure of the $2 \mathrm{D}-\mathrm{HMH}$ film in the vicinity of the $2 \mathrm{D}-\mathrm{HMH} / \mathrm{NiFe}$ interface. When the normal direction of the layered 2D-HMH layer is parallel (antiparallel) to the direction of the $k$ vector of the pump pulse, the $+\mathbf{p}_{\text {dipole }}(z)$ $\left(-\mathbf{p}_{\text {dipole }}(z)\right)$ is formed in the layered structure that is mostly responsible for the control of the forward (backward) $\mathrm{THz}$ radiation.

Whereas the $\mathbf{p}_{\text {dipole }}(z)$ will flip its direction when the $\mathbf{B}$ field reverses $\left(\mathbf{p}_{\text {dipole }}(z) \propto \mathbf{J}_{p}\left(P_{\text {laser }}\right) \times \mathbf{B}\right)$, the momentum shift $\mathbf{P}_{x}$ will be along the same direction regardless of the $\mathbf{B}$ field direction according to Eq. (2) $\left(\propto \mathbf{B} \times \mathbf{p}_{\text {dipole }}(z)\right)$, leading to the fieldindependent momentum shift of the Rashba bands along the $\mathrm{x}$-direction as illustrated in Fig. $5 \mathrm{c}-\mathrm{f}$. Thus, the recorded $\mathrm{THz}$ 
emission contains two transient charge currents generated by the conventional IREE process (i.e., $\mathbf{J}_{c}$ (IREE)) and the additional momentum shift, $\mathbf{J}_{c}\left(\mathbf{P}_{x}\right)$ as described below:

$$
\mathbf{J}_{c}(\mathrm{THz})=\mathbf{J}_{c}(\operatorname{IREE}, \mathbf{B})+\mathbf{J}_{c}\left(\mathbf{P}_{x},|\mathbf{B}|\right)
$$

where $\mathbf{J}_{c}$ (IREE) component is field-dependent that generates symmetric $\mathrm{THz}$ amplitude upon the transient spin current injection. Most intriguingly, however, the $\mathbf{J}_{c}\left(\mathbf{P}_{x}\right)$ component does not change the polarity between two fields, which accounts for the observed asymmetric $\mathrm{THz}$ radiation. As for an arbitrary linear polarization, i.e., the laser pulse rotating away from the $x$-axis, $\mathbf{J}_{p}\left(P_{\text {laser }}\right)$ will come from the projection of light polarization along the $x$-axis, which is consistent with the pump polarization dependence with respect to the applied $\mathbf{B}$ field as shown in Fig. 4.

Summary. Our work shows the realization of ultrafast spin-tocharge conversion in 2D-HMH films with $\mathrm{THz}$ frequencies. It offers promising routes towards interconversion between photons, charges, and spin states using scalable, printable, solutionprocessed hybrid compounds. Taking advantage of layered structures in the presence of the injected superdiffusive spinpolarized current, the phase and intensity of $\mathrm{THz}$ radiation in $2 \mathrm{D}$ $\mathrm{HMH}$ materials can be manipulated by the magnetization state of the adjacent ferromagnet and the pump polarization, offering contactless coherent control of the $\mathrm{THz}$ radiation utilizing spintronic toolkits. Our work will launch a promising testbed for designing a wide variety of low-dimensional $\mathrm{HMH}$ materials for future solution-based hybrid spintronic- $\mathrm{THz}$ emitter applications, bridging the gap between optoelectronics in $\mathrm{HMH}$ materials and $\mathrm{THz}$ spintronics.

\section{Methods}

Device fabrication and characterization. 2D-HMH films were spin-coated on sapphire substrates in a glove box designed for thin-film preparation. The quality of the prepared 2D-HMH films was characterized by XRD and absorption spectroscopy (S.I. Fig. S2), respectively. The surface roughness of films was characterized by AFM (S.I. Fig. S2). Following the spin coating procedure, the prepared 2D$\mathrm{HMH}$ thin films were immediately transferred into a glove-box-integrated deposition chamber without exposure to air. $\mathrm{Ni}_{81} \mathrm{Fe}_{19}$ and $\mathrm{SiO}_{2}$ layers were then deposited at room temperature using e-beam evaporation with a chamber base pressure of $1 \times 10^{-7}$ Torr and evaporation rates of 0.5 and $2.0 \mathrm{~A} / \mathrm{s}$, respectively. The $\mathrm{NiFe}$ layer was deposited on a $4 \times 4 \mathrm{~mm}^{2}$ area of the substrate, while the $\mathrm{SiO}_{2}$ layer was then coated over the entire $5 \times 5 \mathrm{~mm}^{2}$ substrate as a capping layer to protect the $2 \mathrm{D}-\mathrm{HMH}$ thin film during the $\mathrm{THz}$ measurement. Our in-situ device fabrication is the key to successful hybrid THz measurement, which significantly protects the device from being oxidized or degraded during the preparation stage due to potential oxygen and humidity. The control devices, such as Sapphire/NiFe/Pt, Sapphire/Pt/NiFe, Sapphire/2D-HMH/SiO ${ }_{2}$, and Sapphire $/ \mathrm{NiFe} / \mathrm{SiO}_{2}$ were prepared following the same protocol.

THz emission measurements. A THz time-domain emission spectroscopy system in transmission geometry is utilized to study the $\mathrm{THz}$ emission from RD-HMHs system at room temperature. A Ti: Sapphire laser system with $800 \mathrm{~nm}$ center wavelength, $2 \mathrm{kHz}$ repetition rate, and $35 \mathrm{fs}$ pulse duration is used as the laser source. The laser beam is split into a pump beam and a probe beam via a beam splitter. The pump beam with a pump fluence of $\sim 1.0 \mathrm{~mJ} / \mathrm{cm}^{2}$ (see S.I. Fig. S11) is used to normally excite the device, from which the emitted $\mathrm{THz}$ radiation is collected, collimated, and focused onto a ZnTe crystal (300 $\mu$ m-thickness, 110 -cut) by a pair of off-axis parabolic mirrors. A pair of wire-grid $\mathrm{THz}$ polarizers in the frequency range of $0.1-3 \mathrm{THz}$ is used in between the pair of off-axis parabolic mirrors to analyze the $\mathrm{THz}$ polarization. The weak probe beam is focused on the ZnTe crystal, overlapping with the generated $\mathrm{THz}$ pulses spatially and temporally. The transmitted probe beam passes through a quarter-wave plate and a Wollaston prism and then is detected by a balanced photodetector.

The thickness of the used sapphire substrate (C-plane (0001), MTI Corp.) is $0.5 \mathrm{~mm}$. The reported dielectric constant for this substrate is 11.58 along the $c$-axis, corresponding to a refractive index $(n)$ of $\sim 3.4$. The emitted $\mathrm{THz}$ pulse is reflected at the interface between the sapphire substrate and the air and passes the substrate twice, which results in a delayed $\mathrm{THz}$ peak in the detected $\mathrm{THz}$ waveform The total delayed time $\Delta \mathrm{t}$ can be calculated by $(0.5 \mathrm{~mm} \times 2) /(c / n) \approx 11 \mathrm{ps}$, where $c$ is the speed of light in vacuum. The calculated delay time is consistent with our observations.

\section{Data availability}

All data are available in the main text or supplementary materials. Additional data related to this paper may be requested from the authors.

Received: 22 October 2020; Accepted: 14 September 2021; Published online: 30 September 2021

\section{References}

1. Walowski, J. \& Münzenberg, M. Perspective: ultrafast magnetism and THz spintronics. J. Appl. Phys. 120, 140901 (2016).

2. Tonouchi, M. Cutting-edge terahertz technology. Nat. Photonics 1, 97-105 (2007).

3. Dhillon, S. S. et al. The 2017 terahertz science and technology roadmap. J. Phys. D. Appl. Phys. 50, 43001 (2017).

4. Seifert, T. et al. Efficient metallic spintronic emitters of ultrabroadband terahertz radiation. Nat. Photonics 10, 483-488 (2016).

5. Zhou, C. et al. Broadband terahertz generation via the interface inverse Rashba-Edelstein effect. Phys. Rev. Lett. 121, 86801 (2018).

6. Jungfleisch, M. B. et al. Control of terahertz emission by ultrafast spin-charge current conversion at Rashba interfaces. Phys. Rev. Lett. 120, 207207 (2018).

7. Vaswani, C. et al. Terahertz second-harmonic generation from lightwave acceleration of symmetry-breaking nonlinear supercurrents. Phys. Rev. Lett. 124, 207003 (2020).

8. Luo, L. et al. A light-induced phononic symmetry switch and giant dissipationless topological photocurrent in ZrTe5. Nat. Mater. 20, 329-334 (2021).

9. Ando, K. et al. Inverse spin-Hall effect induced by spin pumping in metallic system. J. Appl. Phys. 109, 103913 (2011).

10. Lesne, E. et al. Highly efficient and tunable spin-to-charge conversion through Rashba coupling at oxide interfaces. Nat. Mater. 15, 1261-1266 (2016).

11. Song, Q. et al. Observation of inverse Edelstein effect in Rashba-split 2DEG between $\mathrm{SrTiO}_{3}$ and $\mathrm{LaAlO}_{3}$ at room temperature. Sci. Adv. 3, e1602312 (2017).

12. Sánchez, J. C. R. et al. Spin-to-charge conversion using Rashba coupling at the interface between non-magnetic materials. Nat. Commun. 4, 2944 (2013).

13. Zhang, W., Jungfleisch, M. B., Jiang, W., Pearson, J. E. \& Hoffmann, A. Spin pumping and inverse Rashba-Edelstein effect in $\mathrm{NiFe} / \mathrm{Ag} / \mathrm{Bi}$ and $\mathrm{NiFe} / \mathrm{Ag} / \mathrm{Sb}$. J. Appl. Phys. 117, 17C727 (2015).

14. Wang, J. et al. Spin-optoelectronic devices based on hybrid organic-inorganic trihalide perovskites. Nat. Commun. 10, 129 (2019).

15. Kepenekian, M. et al. Rashba and Dresselhaus effects in hybrid organicinorganic perovskites: from basics to devices. ACS Nano 9, 11557-11567 (2015).

16. Sun, D. et al. Surface-enhanced spin current to charge current conversion efficiency in $\mathrm{CH} 3 \mathrm{NH} 3 \mathrm{PbBr} 3$-based devices. J. Chem. Phys. 151, 174709 (2019).

17. Chen, Z., Guo, Y., Wertz, E. \& Shi, J. Merits and challenges of Ruddlesden-Popper soft halide perovskites in electro-optics and optoelectronics. Adv. Mater. 31, 1803514 (2019).

18. Cao, D. H., Stoumpos, C. C., Farha, O. K., Hupp, J. T. \& Kanatzidis, M. G. 2D homologous perovskites as light-absorbing materials for solar cell applications. J. Am. Chem. Soc. 137, 7843-7850 (2015).

19. Niesner, D. et al. Structural fluctuations cause spin-split states in tetragonal $\left(\mathrm{CH}_{3} \mathrm{NH}_{3}\right) \mathrm{PbI}_{3}$ as evidenced by the circular photogalvanic effect. Proc. Natl Acad. Sci. USA 115, 9509-9514 (2018).

20. Zhai, Y. et al. Giant Rashba splitting in $2 \mathrm{D}$ organic-inorganic halide perovskites measured by transient spectroscopies. Sci. Adv. 3, 2017-2018 (2017).

21. Chanana, A. et al. Colour selective control of terahertz radiation using twodimensional hybrid organic inorganic lead-trihalide perovskites. Nat. Commun. 8, 1328 (2017).

22. Liu, X. et al. Circular photogalvanic spectroscopy of Rashba splitting in $2 \mathrm{D}$ hybrid organic-inorganic perovskite multiple quantum wells. Nat. Commun. 11, 323 (2020).

23. Chanana, A., Liu, X., Zhang, C., Vardeny, Z. V. \& Nahata, A. Ultrafast frequency-agile terahertz devices using methylammonium lead halide perovskites. Sci. Adv. 4, eaar7353 (2018)

24. Stoumpos, C. C. et al. Ruddlesden-Popper hybrid lead iodide perovskite 2D homologous semiconductors. Chem. Mater. 28, 2852-2867 (2016).

25. Eschenlohr, A. et al. Ultrafast spin transport as key to femtosecond demagnetization. Nat. Mater. 12, 332-336 (2013).

26. Wu, Q. \& Zhang, X. -C. Free-space electro-optic sampling of terahertz beams. Appl. Phys. Lett. 67, 3523-3525 (1995).

27. Torosyan, G., Keller, S., Scheuer, L., Beigang, R. \& Papaioannou, E. T. Optimized spintronic terahertz emitters based on epitaxial grown Fe/Pt layer structures. Sci. Rep. 8, 1311 (2018). 
28. Guzelturk, B. et al. Terahertz emission from hybrid perovskites driven by ultrafast charge separation and strong electron-phonon coupling. Adv. Mater. 30, 1704737 (2018)

29. Apostolopoulos, V. \& Barnes, M. E. THz emitters based on the photo-Dember effect. J. Phys. D. Appl. Phys. 47, 374002 (2014).

30. Wright, A. R., Xu, X. G., Cao, J. C. \& Zhang, C. Strong nonlinear optical response of graphene in the terahertz regime. Appl. Phys. Lett. 95, 72101 (2009).

31. Zhang, C. et al. Magnetic field effects in hybrid perovskite devices. Nat. Phys. 11, 427-434 (2015).

32. Takahashi, Y. K., Kasai, S., Hirayama, S., Mitani, S. \& Hono, K. All-metallic lateral spin valves using $\mathrm{Co} 2 \mathrm{Fe}(\mathrm{Ge} 0.5 \mathrm{Ga} 0.5)$ Heusler alloy with a large spin signal. Appl. Phys. Lett. 100, 52405 (2012).

33. Ramos, R. et al. Observation of the spin Seebeck effect in epitaxial Fe3O4 thin films. Appl. Phys. Lett. 102, 072413 (2013).

34. Huisman, T. J. et al. Femtosecond control of electric currents in metallic ferromagnetic heterostructures. Nat. Nanotechnol. 11, 455-458 (2016).

35. Cheng, L. et al. Far out-of-equilibrium spin populations trigger giant spin injection into atomically thin MoS2. Nat. Phys. 15, 347-351 (2019).

36. Liu, Z. et al. Ultrafast control of excitonic Rashba fine structure by phonon coherence in the metal halide perovskite $\mathrm{CH} 3 \mathrm{NH} 3 \mathrm{PbI} 3$. Phys. Rev. Lett. 124, 157401 (2020).

37. Butov, L. V. et al. Observation of magnetically induced effective-mass enhancement of quasi-2D excitons. Phys. Rev. Lett. 87, 216804 (2001).

\section{Acknowledgements}

E.V. and D.S. acknowledge supports from US National Science Foundation, ECCS1933297. W.Z. acknowledges partial supports from Michigan Space Grant Consortium, Air-Force Office of Scientific Research under award no. FA9550-19-1-0254, and US National Science Foundation, ECCS-1933301. L.Y. and W.Y. acknowledge supports from US National Science Foundation, ECCS-1933324, and the Center for Hybrid Organic Inorganic Semiconductors for Energy (CHOISE), an Energy Frontier Research Center funded by the U.S. Department of Energy, Office of Science, Office of Basic Energy Sciences. K.C. and H.W. at Argonne are supported by the U.S. Department of Energy, Office of Science, Materials Science Engineering Division, as well as under contract no. DE-SC0012509. Use of the Center for Nanoscale Materials was supported by the U.S. Department of Energy, Office of Science, Basic Energy Science, under contract no. DEAC02-06CH11357. J.W. and Y.Y. were supported by the Ames Laboratory, the U.S. Department of Energy, Office of Science, Basic Energy Sciences, Materials Science and Engineering Division under Contract No. DE-AC02- 07CH11358. XRD and AFM were performed in part at the Chapel Hill Analytical and Nanofabrication Laboratory, CHANL, a member of the North Carolina Research Triangle Nanotechnology Network, RTNN, which is supported by the US National Science Foundation, Grant ECCS-1542015, as part of the National Nanotechnology Coordinated Infrastructure, NNCI. CD measurement was performed at the UNC Macromolecular Interactions Facility, supported by the National Cancer Institute of the US National Institutes of Health under Award No. P30CA016086. We thank Prof. Yizheng Wu of Fudan University for beneficial discussion.

\section{Author contributions}

D.S., W.Z., and H.W. conceived this study and the experiments. K.C. and H.W. performed $\mathrm{THz}$ emission measurements. E.V. and L.Y. fabricated the devices. L.Y. characterized the 2D-HMH film with XRD, absorption, AFM, and CD spectra. J.W. and Y.Y. were responsible for the model building of the asymmetric $\mathrm{THz}$ emission. Q.Z., Y.L. Y.X., H.Q., R.S., and A.H. assisted in the data discussion. E.V., D.S., and W.Z. wrote the manuscript. H.W., W.Y., W.Z., and D.S. were responsible for the project planning, group managing, and manuscript writing. All authors discussed the results, worked on data analysis and manuscript preparation.

\section{Competing interests}

The authors declare no competing interests.

\section{Additional information}

Supplementary information The online version contains supplementary material available at https://doi.org/10.1038/s41467-021-26011-6.

Correspondence and requests for materials should be addressed to Wei You, Haidan Wen, Wei Zhang or Dali Sun.

Peer review information Nature Communications thanks Evangelos Papaioannou and the other, anonymous, reviewer(s) for their contribution to the peer review of this work Peer reviewer reports are available.

Reprints and permission information is available at http://www.nature.com/reprints

Publisher's note Springer Nature remains neutral with regard to jurisdictional claims in published maps and institutional affiliations.

(c) (i) Open Access This article is licensed under a Creative Commons Attribution 4.0 International License, which permits use, sharing, adaptation, distribution and reproduction in any medium or format, as long as you give appropriate credit to the original author(s) and the source, provide a link to the Creative Commons license, and indicate if changes were made. The images or other third party material in this article are included in the article's Creative Commons license, unless indicated otherwise in a credit line to the material. If material is not included in the article's Creative Commons license and your intended use is not permitted by statutory regulation or exceeds the permitted use, you will need to obtain permission directly from the copyright holder. To view a copy of this license, visit http://creativecommons.org/ licenses/by/4.0/.

(C) The Author(s) 2021 